\title{
A model for the bus system in Cuernavaca (Mexico)
}

\author{
Jinho Baik $^{1}$, Alexei Borodin ${ }^{2}$, Percy Deift ${ }^{3}$ and Toufic Suidan ${ }^{4}$ \\ ${ }^{1}$ Department of Mathematics, University of Michigan, Ann Arbor, MI, USA \\ 2 Department of Mathematics, California Institute of Technology, Pasadena, CA, USA \\ ${ }^{3}$ Courant Institute of Mathematical Sciences, New York University, New York City, NY, USA \\ ${ }^{4}$ Department of Mathematics, University of California, Santa Cruz, CA, USA \\ E-mail: baik@umich.edu, borodin@caltech.edu,deift@cims.nyu.edu and tsuidan@ucsc.edu
}

Received 19 October 2005, in final form 17 January 2006

Published 27 June 2006

Online at stacks.iop.org/JPhysA/39/8965

\begin{abstract}
The bus system in Cuernavaca, Mexico and its connections to random matrix distributions have been the subject of an interesting recent study by M Krbálek and $P$ Šeba in $[15,16]$. In this paper we introduce and analyse a microscopic model for the bus system. We show that introducing a natural repulsion does produce random matrix distributions in natural double scaling regimes. The techniques employed include non-intersecting paths, logarithmic potential theory, determinantal point processes, and asymptotic analysis of several orthogonal polynomial ensembles. In addition, we introduce a circular bus model and include various calculations of non-crossing probabilities.
\end{abstract}

PACS number: $02.10 . Y n$

\section{Introduction}

The bus transportation system in Cuernavaca, Mexico, has certain innovative and distinguishing features and has been the subject of an intriguing recent study by M Krbálek and P Šeba in $[15,16]$. The situation is as follows. We quote from [15]:

In Cuernavaca there is no covering company responsible for organizing the city transport. Consequently, constraints such as a time table that represents external influence on the transport do not exist. Moreover, each bus is the property of the driver. The drivers try to maximize their income and hence the number of passengers they transport. This leads to competition among the drivers and to their mutual interaction. It is known that without additive interaction the probability distribution of the distances between subsequent buses is close to the Poissonian distribution and can be described by the standard bus route model.... A Poisson-like distribution implies, however, that the probability of close encounters of two buses is high (bus clustering) which is in conflict with the effort of the drivers to maximize the number 
of transported passengers and accordingly to maximize the distance to the preceding bus. In order to avoid the unpleasant clustering effect the bus drivers in Cuernavaca engage people who record the arrival times of buses at significant places. Arriving at a checkpoint, the driver receives the information of when the previous bus passed that place. Knowing the time interval to the preceding bus the driver tries to optimize the distance to it by either slowing down or speeding up. In such a way the obtained information leads to a direct interaction between buses ....

In [15], Krbálek and Šeba describe their work in analysing the statistics of bus arrivals on line 4 close to the city centre. They study, in particular, the bus spacing distribution and also the bus number variance measuring the fluctuations of the total number of buses arriving at a fixed location during a time interval $T$. Quite remarkably, Krbálek and Šeba find that these two statistics are well modelled by the Gaussian unitary ensemble (GUE) of random matrix theory (RMT) (see figures 2 and 3 in [15]). Our goal in this paper is to provide a plausible explanation of these observations, and to this end we introduce a microscopic model for the bus line that leads simply and directly to GUE.

As noted in [15] the number variance for the buses is in good agreement with the GUE formula up to a time interval $T=3$ (see figure 3 in [15]). As explained in [15], this behaviour is consonant with the fact that each bus driver, using the information given by the recorder, interacts with the bus immediately behind him and the bus immediately in front of him. In other words, the primary interaction is a three-body interaction. As is well known, particle systems modelled by GUE involve interactions between all the particles. This means, in particular, that if we use GUE to model a system with nearest-neighbour (or more generally, short range) interactions we should restrict our attention to statistics that involve only nearest-neighbour (or short range) interactions. This is the case for the spacing distributions and hence one is able to account for the good agreement between GUE and the observed data for this statistic across the entire parameter range in [15]; this is in contrast to the number variance where, as noted above, there is good agreement only up to $T=3$.

The paper is organized as follows. In section 2, we introduce our model. In section 3, we describe the double scaling limits of interest and indicate how to analyse these limits for our model using standard asymptotic techniques from random matrix theory. Our main results on the spacing distributions, as well as on the number variance, are stated at the end of section 3 . In section 4, we show how to modify the model to include alternative bus schedules.

Our analysis is based on the fact that certain nonintersecting path models lead to random matrix type ensembles, more exactly, orthogonal polynomial ensembles. Various manifestations of this phenomenon can be observed in [3, 10, 13, 14].

Finally, in view of the calculations that follow, we note that formula (4) in [15] for the density of the spacing distribution is the so-called 'Wigner surmise' rather than the Gaudin distribution. The Gaudin distribution (see (28)) gives the exact formula for the spacing distribution: However, the Wigner surmise is known to approximate this exact formula to high accuracy (see Mehta [18]).

\section{Basic model}

We introduce a 'bare-bones' model which captures the two salient features of the bus system:

(a) the stop-start motion of the buses, and

(b) the fact that the buses effectively repel each other, on the basis of information from the recorders. 


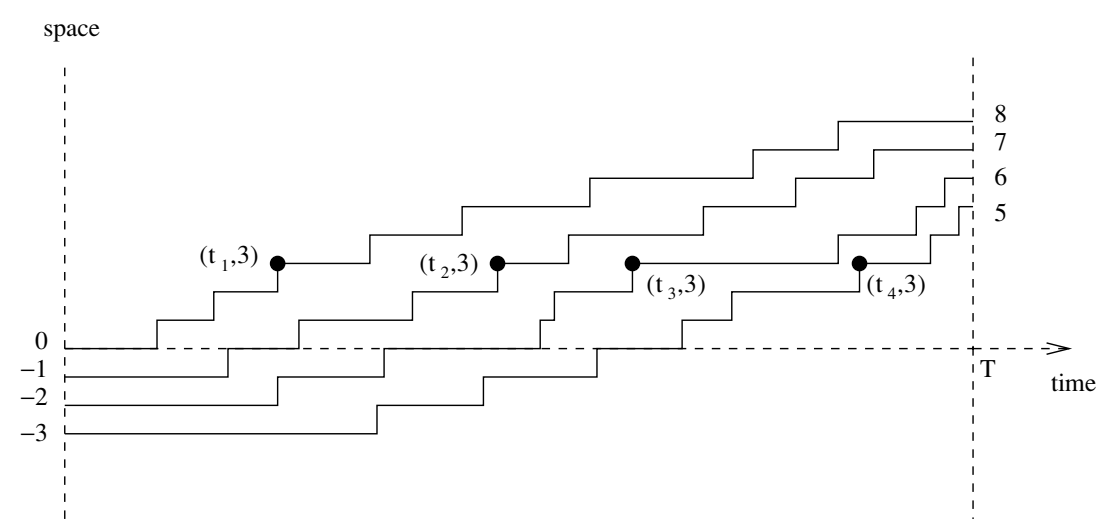

Figure 1. Conditioned Poisson walks arriving at location 3.

The dynamics of our bus model takes place on the lattice $\mathbb{Z}$ as follows. Assume that there are $n$ buses. Fix $N>n$ and $T>0: N$ denotes the end of the line which is reached by time $T$. At time $t=0$ the $i$ th bus is at location $1-i, 1 \leqslant i \leqslant n$. The evolution of the bus process, $L_{t}=\left(L_{t}^{1}, \cdots, L_{t}^{n}\right)$, is given by $n$ independent rate 1 Poisson processes conditioned not to intersect for $t \in[0, T]$, and subject to the terminal condition $L_{\mathrm{T}}=(N, N-1, \ldots, N-n+1)$. Consider buses arriving at a fixed point $x \in\{1,2, \ldots, N-n+1\}$ with consecutive arrival times $0<t_{1}<\cdots<t_{k}<T$ (see figure 1). by

The probability density for the arrival times $t_{1}, \ldots, t_{n}, A_{x}\left(t_{1}, \ldots, t_{n}\right) \mathrm{d} t_{1} \cdots \mathrm{d} t_{n}$, is given $\frac{\mathbb{P}\left[\{(0,1-i)\} \rightarrow\left\{\left(t_{i}, x-1\right)\right\} ; \text { no intersection }\right] \mathbb{P}\left[\left\{\left(t_{i}, x\right)\right\} \rightarrow\{(T, N+(1-i))\} ; \text { no intersection }\right] \mathrm{d} t_{1} \cdots \mathrm{d} t_{n}}{\mathbb{P}[\{(0,1-i)\} \rightarrow\{(T, N+(1-i))\} ; \text { no intersection }]}$.

The fact that the numerator in (1) splits into a product of two factors is a consequence of the strong Markov property of $L_{t}$ : in particular, functionals of the parts of the paths $\left\{\left(t_{i}, x\right) \rightarrow(T, N-(1-i)), 1 \leqslant i \leqslant n\right\}$ depend only on the increments of the process after some stopping times and hence, are independent of any functionals of the initial parts $\left\{(0,1-i) \rightarrow\left(t_{i}, x-1\right), 1 \leqslant i \leqslant n\right\}$ of the paths. Note also that the endpoints $\left(t_{i}, x-1\right)$, $1 \leqslant i \leqslant n$, reflect the fact that the $i$ th path jumps at $t_{i}$, and not before. Similar dynamical models with $T=\infty$ were considered in [14].

As we now show, each term in ratio (1) has a determinantal form of Karlin-McGregor type [11].

\section{Lemma 1.}

$\mathbb{P}\left[\{(0,1-i)\} \rightarrow\left\{\left(t_{i}, x-1\right)\right\} ;\right.$ no intersection $]=\operatorname{det}\left(\mathrm{e}^{-t_{j}} \frac{t_{j}^{x+i-2}}{(x+i-2) !}\right)_{i, j=1}^{n}$,

$\mathbb{P}\left[\left\{\left(t_{i}, x\right)\right\} \rightarrow\{(T, N+(1-i))\} ;\right.$ no intersection $]=\operatorname{det}\left(\mathrm{e}^{-\left(T-t_{j}\right)} \frac{\left(T-t_{j}\right)^{N+(1-i)-x}}{(N+(1-i)-x) !}\right)_{i, j=1}^{n}$

$\mathbb{P}[\{(0,1-i)\} \rightarrow\{(T, N+(1-i))\} ;$ no intersection $]=\operatorname{det}\left(\mathrm{e}^{-T} \frac{T^{N+i-j}}{(N+i-j) !}\right)_{i, j=1}^{n}$. 
Proof. Since the proofs of (2) and (3) are similar, we only prove (2). The proof is a variation on the standard Karlin-McGregor argument [11]. Fix $0<t_{1}<\cdots<t_{n}<T$ and define the following stopping times:

$$
\begin{aligned}
\tau_{1}= & {\left[\inf \left\{s \in\left[0, t_{1}\right]: L_{s}^{1}=L_{s}^{2}\right\}\right] \wedge T } \\
\tau_{2}= & {\left[\inf \left\{s \in\left[0, t_{2}\right]: L_{s}^{2}=L_{s}^{3}\right\}\right] \wedge T } \\
& \cdots \\
\tau_{n-1}= & {\left[\inf \left\{s \in\left[0, t_{n-1}\right]: L_{s}^{n-1}=L_{s}^{n}\right\}\right] \wedge T . }
\end{aligned}
$$

Observe that

$$
\begin{aligned}
& \operatorname{det}\left(\mathrm{e}^{-t_{j}} \frac{t_{j}^{x+i-2}}{(x+i-2) !}\right)_{i, j=1}^{n}=\sum_{\sigma \in S_{n}} \operatorname{sgn}(\sigma) \prod_{i=1}^{n} \mathbb{P}\left(L_{t_{i}}^{\sigma(i)}=x-1\right) \\
&=\sum_{\sigma \in S_{n}} \operatorname{sgn}(\sigma) \mathbb{E}\left(\prod_{i=1}^{n} \mathbb{1}_{\left\{L_{t_{i}}^{\sigma(i)}=x-1\right\}}\right) \\
&=\sum_{\sigma \in S_{n}} \operatorname{sgn}(\sigma) \mathbb{E}\left\{\left[\prod_{i=1}^{n} \mathbb{1}_{\left\{L_{t_{i}}^{\sigma(i)}=x-1\right\}}\right]\left[\mathbb{1}_{\tau_{1} \wedge \cdots \wedge \tau_{n-1}=T}+\sum_{j=1}^{n-1} \mathbb{1}_{\tau_{1} \wedge \cdots \wedge \tau_{n-1}=\tau_{j}<T}\right]\right\} \\
&=\sum_{\sigma \in S_{n}} \operatorname{sgn}(\sigma) \mathbb{E}\left[\mathbb{1}_{\tau_{1} \wedge \cdots \wedge \tau_{n-1}=T} \prod_{i=1}^{n} \mathbb{1}_{\left\{L_{t_{i}}^{\sigma(i)}=x-1\right\}}\right] \\
&+\sum_{j=1}^{n-1} \sum_{\sigma \in S_{n}} \operatorname{sgn}(\sigma) \mathbb{E}\left[\left(\mathbb{1}_{\tau_{1} \wedge \cdots \wedge \tau_{n-1}=\tau_{j}<T}\right) \prod_{i=1}^{n} \mathbb{1}_{\left\{L_{t_{i}}^{\sigma(i)}=x-1\right\}}\right] \\
&= \mathbb{P}\left[\{(0,1-i)\} \rightarrow\left\{\left(t_{i}, x-1\right)\right\} ; \text { no intersection }\right] \\
&+\sum_{j=1}^{n-1} \sum_{\sigma \in S_{n}} \operatorname{sgn}(\sigma) \mathbb{E}\left[\left(\mathbb{1}_{\tau_{1} \wedge \cdots \wedge \tau_{n-1}=\tau_{j}<T}\right) \prod_{i=1}^{n} \mathbb{1}_{\left\{L_{t_{i}}^{\sigma(i)}=x-1\right\}}\right],
\end{aligned}
$$

since the only nonzero term in the first sum is the term corresponding to the identity permutation. We need only show that the second summation vanishes. Let $\rho_{j} \in S_{n}$ be the transposition $(j, j+1)$. By the strong Markov property for $L_{t}$,

$$
\begin{array}{r}
\operatorname{sgn}(\sigma) \mathbb{E}\left[\left(\mathbb{1}_{\tau_{1} \wedge \cdots \wedge \tau_{n-1}=\tau_{j}<T}\right) \prod_{i=1}^{n} \mathbb{1}_{\left\{L_{t_{i}}^{\sigma(i)}=x-1\right\}}\right]+\operatorname{sgn}\left(\rho_{j} \sigma\right) \\
\quad \times \mathbb{E}\left[\left(\mathbb{1}_{\tau_{1} \wedge \cdots \wedge \tau_{n-1}=\tau_{j}<T}\right) \prod_{i=1}^{n} \mathbb{1}_{\left\{L_{t_{i}}^{\rho_{j} \sigma(i)}=x-1\right\}}\right]=0 .
\end{array}
$$

Since for each $j$ the action of the given transposition $\rho_{j}$ is an involution on $S_{n}$,

$$
\sum_{\sigma \in S_{n}} \operatorname{sgn}(\sigma) \mathbb{E}\left[\left(\mathbb{1}_{\tau_{1} \wedge \cdots \wedge \tau_{n-1}=\tau_{j}<T}\right) \prod_{i=1}^{n} \mathbb{1}_{\left\{L_{t_{i}}^{\sigma(i)}=x-1\right\}}\right]=0 .
$$

This immediately implies that the second sum in equation (5) vanishes leaving only the desired probability. 
The proof of the third equality (4) is just the standard Karlin-McGregor argument [11]: in the proof of (2), simply replace the $\tau_{i}$ 's by

$$
\tilde{\tau}_{i}=T \wedge \inf \left\{s \in[0, T]: L_{s}^{i}=L_{s}^{i+1}\right\}, \quad \text { for } \quad i=1, \ldots, n-1,
$$

$x-1$ by $N+(1-i)$, and set $t_{i}=T$ for $1 \leqslant i \leqslant n$. This completes the proof of the lemma (1).

The determinants in (2) and (3) are multiples of Vandermonde determinants. Indeed, using multilinearity of the determinant one shows that

$$
\begin{gathered}
\operatorname{det}\left(\mathrm{e}^{-t_{j}} \frac{t_{j}^{x+i-2}}{(x+i-2) !}\right)_{i, j=1}^{n}=\frac{\mathrm{e}^{-\sum_{j=1}^{n} t_{j}}}{\prod_{i=1}^{n}(x+i-2) !} \prod_{j=1}^{n} t_{j}^{x-1} \prod_{1 \leqslant i<j \leqslant n}\left(t_{j}-t_{i}\right) \\
\operatorname{det}\left(\mathrm{e}^{-\left(T-t_{j}\right)} \frac{\left(T-t_{j}\right)^{N+(1-i)-x}}{(N+(1-i)-x) !}\right)_{i, j=1}^{n}=\frac{\mathrm{e}^{-\sum_{j=1}^{n}\left(T-t_{j}\right)}}{\prod_{i=1}^{n}(N+1-x-i) !} \\
\times \prod_{j=1}^{n}\left(T-t_{j}\right)^{N-x+1-n} \prod_{1 \leqslant i<j \leqslant n}\left(t_{j}-t_{i}\right) .
\end{gathered}
$$

Inserting (4), (7) and (8) into (1) and setting $\tilde{y}_{j}=\frac{t_{j}}{T}$, one obtains

$$
\begin{aligned}
& A\left(t_{1}, \ldots, t_{n}\right) \mathrm{d} t_{1} \cdots \mathrm{d} t_{n}=\tilde{A}\left(\tilde{y}_{1}, \ldots, \tilde{y}_{n}\right) \mathrm{d} \tilde{y}_{1} \cdots \mathrm{d} \tilde{y}_{n} \\
& =C_{N, n, x} \prod_{i<j}\left(\tilde{y}_{j}-\tilde{y}_{i}\right)^{2} \prod_{j=1}^{n} \tilde{y}_{j}^{x-1}\left(1-\tilde{y}_{j}\right)^{N-x-(n-1)} \mathrm{d} \tilde{y}_{1} \cdots \mathrm{d} \tilde{y}_{n},
\end{aligned}
$$

where $C_{N, n, x}=\left(\prod_{i=1}^{n} \frac{1}{(x+i-2) !(N-x+(1-i)) !}\right)\left(\operatorname{det}\left(\frac{1}{(N+i-j) !}\right)_{i, j=1}^{n}\right)^{-1}$. The simple variable change $y_{j}=2 \tilde{y}_{j}-1$ induces the standard Jacobi weights on $[-1,1]$ :

$$
\tilde{C}_{N, n, x} \prod_{i<j}\left(y_{j}-y_{i}\right)^{2} \prod_{j=1}^{n}\left(1+y_{j}\right)^{x-1}\left(1-y_{j}\right)^{N-x-(n-1)} \mathrm{d} y_{1} \cdots \mathrm{d} y_{n},
$$

where $\tilde{C}_{N, n, x}=2^{-n N} C_{N, n, x}$. It is at this point that contact is made with RMT: if we view the $y_{j}$ 's as eigenvalues of an $n \times n$ Hermitian matrix, then (10) is precisely the joint probability density for the eigenvalues of matrices in the so-called Jacobi ensemble. More precisely, for $n \times n$ Hermitian matrices $\left\{M=M^{*}\right\}$, set

$$
w(M)=\operatorname{det}(1+M)^{x-1} \operatorname{det}(1-M)^{N-x-n+1} \operatorname{det}\left(\chi_{(-1,1)}(M)\right),
$$

where $\chi_{(-1,1)}(M)$ is interpreted in terms of the standard functional calculus. Note that $w(M)$ is invariant under unitary conjugation of $M, M \rightarrow U M U^{*}$, for all unitary $U$. Then $P_{n}(M) \mathrm{d} M \equiv \frac{1}{Z_{n}} w(M) \mathrm{d} M$ defines the Jacobi unitary ensemble, where $\mathrm{d} M=$ $\prod_{i=1}^{n} \mathrm{~d} M_{i i} \prod_{i<j} \mathrm{~d} M_{i j}^{R} \prod_{i<j} \mathrm{~d} M_{i j}^{I}$ and $Z_{n}$ is a normalization constant (here $M_{i j}^{R}$ and $M_{i j}^{I}$ denote the real and imaginary parts of the $i j$ entry of $M$ ). A standard calculation (e.g. Mehta [18]) then shows that the distribution function for the eigenvalues, $\left\{\lambda_{i}\right\}$, of matrices in the ensemble is given by (10) with the identification $y_{i}=\lambda_{i}$. In summary, we see from (10) that for finite $N, n$ the arrival times for the buses at a location $x$ are distributed like the eigenvalues of a random matrix from the Jacobi ensemble. By techniques which are now routine in random matrix theory (see [18]), (10) is amenable to asymptotic analysis in certain double scaling limits. These limits (see section 3) correspond to GUE: the fact that Jacobi Unitary Ensemble $\rightarrow$ GUE in the double scaling limit is a particular example of the well-known phenomenon of universality in RMT (see $[6,5,17])$. 
In the previous calculations we considered the arrival times $t_{1}, \ldots, t_{n}$ for the buses at a fixed location, $x$. It is also of interest to consider the locations $x_{1}, \ldots, x_{n}$ at a fixed recording time $t$. It turns out that the distribution of the $x_{i}$ 's is again given by a random matrix-type ensemble, the Krawtchouk unitary ensemble. In [14], König, O'Connell and Roch discovered a similar relation between the Krawtchouk unitary ensemble and Poisson random walks conditioned not to intersect for all positive times. In analogy to (1), we have that the probability $\mathcal{L}_{t}\left(x_{1}, \ldots, x_{n}\right)$ for the positions $x_{1}>\cdots>x_{n}$ of the buses at time $t \in(0, T)$ is given by

$\frac{\mathbb{P}\left(\left\{(0,1-i) \rightarrow\left(t, x_{i}\right)\right\} ; \text { no intersection }\right) \mathbb{P}\left(\left\{\left(t, x_{i}\right) \rightarrow(T, N+1-i)\right\} ; \text { no intersection }\right)}{\mathbb{P}(\{(0,1-i) \rightarrow(T, N+1-i)\} ; \text { no intersection })}$,

and by a Karlin-McGregor argument, similar to and even simpler than that in lemma 1, we obtain the following:

\section{Lemma 2}

$\mathbb{P}\left(\left\{(0,1-i) \rightarrow\left(t, x_{i}\right)\right\} ;\right.$ no intersection $)=\operatorname{det}\left(\mathrm{e}^{-t} \frac{t^{x_{j}+i-1}}{\left(x_{j}+i-1\right) !}\right)_{i, j=1}^{n}$,

$\mathbb{P}\left(\left\{\left(t, x_{i}\right) \rightarrow(T, N+1-i)\right\} ;\right.$ no intersection $)=\operatorname{det}\left(\mathrm{e}^{(T-t)} \frac{(T-t)^{N+1-i-x_{j}}}{\left(N+1-i-x_{j}\right) !}\right)_{i, j=1}^{n}$,

$\mathbb{P}(\{(0,1-i) \rightarrow(T, N+1-i)\} ;$ no intersection $)=\operatorname{det}\left(\mathrm{e}^{-T} \frac{T^{N+i-j}}{(N+i-j) !}\right)_{i, j=1}^{n}$.

Inserting (13), (14) and (15) into (12) we are led to the following formula for $\mathcal{L}_{t}\left(x_{1}, \ldots, x_{n}\right)$ [10]:

$$
\begin{aligned}
\mathcal{L}_{t}\left(x_{1}, \ldots, x_{n}\right)= & {[(N+n-1) !]^{-n}\left[\prod_{i=1}^{n} \frac{(N+i-1) !}{(i-1) !}\right]\left(p-p^{2}\right)^{-\frac{n(n-1)}{2}} \prod_{i<j}\left(y_{j}-y_{i}\right)^{2} } \\
& \times \prod_{j=1}^{n} \frac{(N+n-1) !}{y_{j} !\left(N+n-1-y_{j}\right) !} p^{y_{j}}(1-p)^{N+n-1-y_{j}},
\end{aligned}
$$

where $y_{j}=x_{j}+n-1, p=\frac{t}{T}$ and $N+n-1 \geqslant y_{1}>\cdots y_{n} \geqslant 0$. We have used the elementary identity

$$
\left[\operatorname{det}\left(\frac{1}{(N+i-j) !}\right)_{i, j=1}^{n}\right]^{-1}=\prod_{i=1}^{n} \frac{(N+i-1) !}{(i-1) !}
$$

to derive (16). As indicated above, (16) corresponds to the Krawtchouk unitary ensemble.

An essential aspect in the analysis of the random particle ensembles (10) and (16) is that they are determinantal, i.e. for each $r=1,2, \ldots$, the $r$ th correlation function for the ensemble can be expressed in determinantal form $\operatorname{det}\left(K\left(x_{i}, x_{j}\right)\right)_{i, j=1}^{r}$ for some appropriate correlation kernel $K(x, y)$ (see, for example, [2, 4, 18, 21]). It turns out that the fixed time distribution (16), in particular, can be extended to a dynamical random particle system which is also determinantal. Indeed, fix $k$ times $0<t_{1}<\cdots<t_{k}<T$ and let $x^{(j)}=\left\{x_{1}^{(j)}, \ldots, x_{n}^{(j)}\right\}, 1 \leqslant j \leqslant k$, denote the positions of the buses at times $t_{j}, 1 \leqslant j \leqslant k$. Let $p_{j}=\frac{t_{j}}{T}, 1 \leqslant j \leqslant k$. Then, using the Markov property of the bus system as above, we arrive 
at the following distribution for $x^{(1)}, \ldots, x^{(k)}$ :

$$
\begin{aligned}
\operatorname{Prob}\left[x^{(1)}, \ldots,\right. & \left.x^{(k)}\right]=\mathrm{const} \cdot \prod_{i<j}\left(x_{i}^{(1)}-x_{j}^{(1)}\right) \prod_{i=1}^{n} \frac{p_{1}^{x_{i}^{(1)}}}{x_{1}^{(1)} !} \\
& \times \operatorname{det}\left(V_{p_{2}-p_{1}}\left(x_{i}^{(1)}, x_{j}^{(2)}\right)\right) \operatorname{det}\left(V_{p_{3}-p_{2}}\left(x_{i}^{(2)}, x_{j}^{(3)}\right)\right) \cdots \operatorname{det}\left(V_{p_{k}-p_{k-1}}\left(x_{i}^{(k-1)}, x_{j}^{(k)}\right)\right) \\
& \times \prod_{i<j}\left(x_{i}^{(k)}-x_{j}^{(k)}\right) \prod_{i=1}^{n} \frac{\left(1-p_{k}\right)^{N+n-1-x_{j}^{(k)}}}{\left(N+n-1-x_{j}^{(k)}\right) !}
\end{aligned}
$$

where $V_{p}(x, y)=\frac{p^{y-x}}{(y-x) !}$ if $y \geqslant x$ and $V_{p}(x, y)=0$ otherwise. Using a variant of the Eynard-Mehta theorem [8] as described in [3], it follows directly that (18) is determinantal with an appropriate correlation kernel $K_{t_{i}, t_{j}}(x, y)$. Further analysis yields the following block integral representation for the kernel: for $x \in x^{(i)}, y \in x^{(j)}$,

$$
K_{t_{i}, t_{j}}(x, y)=\frac{1}{(2 \pi \mathrm{i})^{2}} \oint \oint \frac{\left(p_{j}-\left(1-p_{j}\right) t\right)^{y}(1+t)^{N+n-1-y} t^{-n}}{\left(p_{i}-\left(1-p_{i}\right) s\right)^{x+1}(1+s)^{N+n-x} s^{-n}} \frac{\mathrm{d} t}{t-s},
$$

where the integration contours are chosen as follows: $s$ runs along a simple positively oriented contour which goes around $\frac{p_{i}}{1-p_{i}}$ and does not contain $-1 ; t$ runs along a simple positively oriented contour which goes around 0 ; for $p_{i} \geqslant p_{j}$ the $s$-contour contains the $t$-contour and for $p_{i}<p_{j}$ the $s$-contour lies inside the $t$-contour.

In the following section, we will analyse (10) and (16) asymptotically as $N, n, x, t$, $T \rightarrow \infty$. We plan to present the derivation and asymptotic analysis of (18) in a later publication.

\section{The double scaling limits}

We will first describe the arrival statistics of the buses (10) in the limit $N, n, x \rightarrow \infty$ where $\frac{n}{N} \rightarrow v, \frac{x-1}{N} \rightarrow \eta, 0<v, \eta<1$ and $v+\eta<1$. This scaling limit is the natural one from both the physical and mathematical point of view: the number of buses, $n$, should be proportional to the length of the bus route, $N+n$, and the arrival point, $x$, should not be too close to the beginning or the end of the route. The main result is that in the 'unfolded' scale (see below), the Christoffel-Darboux kernel (equivalently, the two-point correlation function) $K_{N, n, x}$ below, converges as $N \rightarrow \infty, \frac{n}{N} \rightarrow \nu, \frac{x}{N} \rightarrow \eta$ to the so-called sine kernel (see below), which is universal in random matrix theory. The proof of the convergence $K_{N, n, x} \rightarrow \mathcal{K}_{\infty}$ for the Jacobi ensemble was given first by Nagao and Wadati [19].

In RMT the analysis of the spacing distribution proceeds via the analysis of the gap distribution (see, for example, [18, 7]):

$$
\mathbb{P}_{N, n}([c, d])=\operatorname{Prob}\left(y_{j} \notin[c, d], i=1, \ldots, n\right),
$$

There are three steps involved in the analysis of $\mathbb{P}_{N, n}([c, d])$ : (a) the first step is to express $\mathbb{P}_{N, n}([c, d])$ in Fredholm determinantal form,

$$
\mathbb{P}_{N, n}([c, d])=\operatorname{det}(I-K)_{L^{2}[c, d]},
$$

for some kernel operator $K$ expressed in terms of certain appropriate orthogonal polynomials (see, in particular, Tracy and Widom [22]); (b) the second step of the analysis is to determine the appropriate scalings for $c$ and $d$; this is accomplished by evaluating the so-called equilibrium measure for the associated logarithmic potential theoretic problem [7, 20]; (c) the third step involves a detailed asymptotic analysis of the appropriate associated orthogonal polynomials in the double scaling limit. 
(a) For $j=1,2, \ldots$, let $p_{j}^{N, n, x}(y)=\gamma_{j}^{N, n, x} y^{j}+\cdots, \gamma_{j}^{N, n, x}>0$ denote the Jacobi polynomials obtained by orthonormalizing $\left\{1, y, y^{2}, \ldots\right\}$ with respect to the weight $w_{N, n, x}(y)=(1-y)^{N-(n+x-1)}(1+y)^{x-1} \chi_{[-1,1]}(y)$. Set $\phi_{j}^{N, n, x}(y)=p_{j}^{N, n, x}(y) w_{N, n, x}^{\frac{1}{2}}(y)$, for $j=1,2, \ldots$. Then, the probability that there is no bus arrival in the interval of time $\left[a_{N, n, x}, b_{N, n, x}\right], \mathbb{P}_{N, n}\left(\left[a_{N, n, x}, b_{N, n, x}\right]\right)$, is given by

$$
\operatorname{det}\left(I-K_{N, n, x}\right)_{L^{2}\left(\left[a_{N, n, x}, b_{N, n, x}\right]\right)},
$$

where

$$
K_{N, n, x}\left(z, z^{\prime}\right)=l_{n} \frac{\phi_{n}^{N, n, x}(z) \phi_{n-1}^{N, n, x}\left(z^{\prime}\right)-\phi_{n}^{N, n, x}\left(z^{\prime}\right) \phi_{n-1}^{N, n, x}(z)}{z-z^{\prime}},
$$

and $l_{n}$ is given by the formula $\frac{k_{n}}{k_{n+1}} \sqrt{\frac{h_{n+1}}{h_{n}}}$ where $h_{n}$ and $k_{n}$ are constants arising in the three term recurrence relation for general orthogonal polynomials; for the Jacobi polynomials, $h_{n}$ and $k_{n}$ are given, respectively, by formulae 22.2.1 and 22.3.1 of Abramowitz and Stegun [1] in the case $\alpha=N-(n+x-1)$ and $\beta=x-1$.

(b) By standard methods (see Saff and Totik [20] and Deift [7]), the equilibrium measure can be determined explicitly for the problem at hand. It turns out that the support of the equilibrium measure is an interval $[a, b] \subset[-1,1]$, and for fixed $\nu, \eta>0$ satisfying $\nu+\eta<1$, the measure takes the form,

$$
\psi(x) \mathrm{d} x=\frac{\eta \sqrt{(x-a)(b-x)}}{\pi \sqrt{(1+a)(1+b)}\left(1-x^{2}\right)} \mathrm{d} x,
$$

where $a$ and $b$ satisfy the relations,

$$
\begin{aligned}
& \frac{\eta}{\sqrt{(1+a)(1+b)}}=-\frac{(\eta+v-1)}{\sqrt{(1-a)(1-b)}}, \\
& (1+v)=\frac{\eta}{\sqrt{(1+a)(1+b)}}-\frac{(\eta+v-1)}{\sqrt{(1-a)(1-b)}},
\end{aligned}
$$

and the radicals represent the positive square root. In the symmetric case where $\eta=\frac{1-v}{2}$,

$$
b=-a=\left(1-\left(\frac{1-v}{1+v}\right)^{2}\right)^{\frac{1}{2}}
$$

(c) Let $\tau_{0} \in(a, b)$. A standard calculation in RMT shows that the expected number of particles per unit interval in a neighbourhood of $\tau_{0} \approx n \psi\left(\tau_{0}\right)$. Thus, changing scales $t \mapsto s:=n \psi\left(\tau_{0}\right) t$, we see that the expected number of particles per unit $s$-interval is 1: this process of rescaling is known as 'unfolding' the data. The probability that there are no bus arrivals in $\left[\tau_{0}-\frac{s}{\psi\left(\tau_{0}\right) n}, \tau_{0}+\frac{s}{\psi\left(\tau_{0}\right) n}\right]$ becomes

$$
\operatorname{det}\left(I-\mathcal{K}_{n}\right)_{L^{2}[-s, s]} \text {, }
$$

where

$\mathcal{K}_{n}\left(\tau_{0}, \xi, \rho\right)=l_{n} \frac{\phi_{n}^{N, n, x}\left(\tau_{0}+\frac{\xi}{\psi\left(\tau_{0}\right) n}\right) \phi_{n-1}^{N, n, x}\left(\tau_{0}+\frac{\rho}{\psi\left(\tau_{0}\right) n}\right)-\phi_{n}^{N, n, x}\left(\tau_{0}+\frac{\rho}{\psi\left(\tau_{0}\right) n}\right) \phi_{n-1}^{N, n, x}\left(\tau_{0}+\frac{\xi}{\psi\left(\tau_{0}\right) n}\right)}{\xi-\rho}$.

Analysing the asymptotics of the Jacobi polynomial as $N, n, x \rightarrow \infty$ as above, we finally see that

$$
\operatorname{det}\left(I-\mathcal{K}_{n}\right)_{L^{2}[-s, s]} \rightarrow \operatorname{det}\left(I-\mathcal{K}_{\infty}\right)_{L^{2}[-s, s]},
$$

where $\mathcal{K}_{\infty}(\xi, \rho)=\frac{\sin \pi(\xi-\rho)}{\pi(\xi-\rho)}$, the so-called sine kernel. To compute the limiting conditional probability that given a bus arrival at time $s$, the next bus arrives at time $t$ we simply compute 
(see, e.g., [22])

$$
\lim _{N \rightarrow \infty} \frac{1}{\psi(s)} \frac{\partial^{2}}{\partial s \partial t} \operatorname{det}\left(I-\mathcal{K}_{N}\right)_{L^{2}[s, t]}=\frac{1}{\psi(s)} \frac{\partial^{2}}{\partial s \partial t} \operatorname{det}\left(I-\mathcal{K}_{\infty}\right)_{L^{2}[s, t]} .
$$

Formula (28), the Gaudin distribution, gives the exact spacing distribution of RMT; as noted in the introduction, this distribution in known to be well approximated by the Wigner surmise used by Krbálek and Šeba [15] to analyse their observations of the bus arrival times.

In addition, using the fact that $K_{N, n, x} \rightarrow K_{\infty}$ in the unfolded scale, one easily sees that the number variance, $H_{N, n, x}(\cdot)$, converges, i.e.

$$
H_{\infty}(s):=\lim _{N \rightarrow \infty, \frac{n}{N} \rightarrow \nu, \frac{x-1}{N} \rightarrow \eta} H_{N, n, x}\left(\frac{s}{n \psi(0)}\right)
$$

exists. Moveover, $H_{\infty}(s)$ is precisely the number variance for GUE, as given, for example, in formula (16.1.3) in [18]. As $s$ becomes large, $H_{\infty}(s) \sim \frac{1}{(\pi)^{2}}(\log (2 \pi s)+\gamma+1)$ where $\gamma$ is Euler's constant, as noted in formula (5) of [15].

The analysis of the fixed time bus locations (16) as $N, T, t \rightarrow \infty, \frac{t}{T} \rightarrow C_{1}, \frac{n}{N} \rightarrow C_{2}$ and $\frac{T}{N} \rightarrow C_{3}$ is very similar and follows the same general procedure (a), (b), (c) above. We note that the Krawtchouk ensemble was first analysed in the same scaling limit by Johansson in his analysis of the Aztec diamond [10]. Again, one finds that in the limit the statistics of the bus locations is governed by the sine kernel.

\section{The circular bus route}

In the previous bus model, we considered the buses moving from one terminal at $t=0$ to the second terminal at time $t=T$. In the final section, we consider a bus model on a circular route. König, O'Connell and Roch (see again [14]) investigated a related model on the discrete circle. Let $\mathbb{Z}_{M}$ be the discrete circle with nodes labelled by $\{0,1,2, \ldots, M-1\}$. We will consider the case of $k<M$ buses travelling along $\mathbb{Z}_{M}$. The buses start at positions $0 \leqslant N_{1}(0)<\cdots<N_{k}(0) \leqslant M-1$ and evolve as independent Poisson processes conditioned not to intersect. Let $\theta, \tilde{\theta} \in \mathbb{Z}_{M}$ and note that the transition probability on $\mathbb{Z}_{M}$ for a single rate 1 Poisson process to travel from state $\theta$ to $\tilde{\theta}$ is

$$
p_{t}(\theta, \tilde{\theta})=\mathrm{e}^{-t} \sum_{l=-\infty}^{\infty} \frac{t^{\tilde{\theta}-\theta+l M}}{\Gamma(\tilde{\theta}-\theta+l M+1)},
$$

where $\Gamma(z)$ is the Gamma function. Let $\left(\theta_{1}, \ldots, \theta_{k}\right)$ be a $k$-tuple of distinct elements of $\mathbb{Z}_{M}$ such that $0 \leqslant \theta_{1}<\cdots<\theta_{k} \leqslant M-1$. Let $\left(\tilde{\theta}_{1}, \ldots, \tilde{\theta}_{k}\right)$ be another $k$-tuple of distinct elements of $\mathbb{Z}_{M}$ such that there exists a cyclic permutation $\sigma \in S_{k}$ for which $\left(\tilde{\theta}_{\sigma(1)}, \ldots, \tilde{\theta}_{\sigma(k)}\right)$ satisfies $0 \leqslant \tilde{\theta}_{\sigma(1)}<\cdots<\tilde{\theta}_{\sigma(k)} \leqslant M-1$. Given that the buses begin at positions $\theta_{1}<\cdots<\theta_{k}$ at time 0 , the probability that they are at locations $\tilde{\theta}_{1}, \ldots, \tilde{\theta}_{k}$ at time $t$, and have not intersected in the mean time, is given by the determinantal expression

$$
\operatorname{det}\left(p_{t}\left(\theta_{i}, \tilde{\theta}_{j}\right)\right)_{i, j=1}^{k} \text {. }
$$

We prove this expression by adapting an alternate proof of the Karlin-McGregor formula from the line to the circle as follows.

Let $\mathbb{Z}_{M}^{k}$ be defined as the subset of $\mathbb{Z}^{k} / M \mathbb{Z}^{k}$ for which there exists a cyclic permutation $\sigma \in S_{k}$ such that $0 \leqslant z_{\sigma(1)} \leqslant z_{\sigma(2)} \leqslant \cdots \leqslant z_{\sigma(k)} \leqslant M-1$. For $f: \mathbb{Z}_{M \geqslant}^{k} \rightarrow \mathbb{R}$, define the operator

$L f(z)=\sum_{i=1}^{k}\left[f\left(z_{1}, \ldots, z_{i}+1, \ldots, z_{k}\right)-f(z)\right], \quad$ for $\quad z \in \mathbb{Z}_{M \geqslant}^{k} \backslash \partial \mathbb{Z}_{M \geqslant}^{k}$ 
and

$$
L f(z)=0 \quad \text { if } \quad z \in \partial \mathbb{Z}_{M \geqslant}^{k} .
$$

As we will see, in order to prove the circular Karlin-McGregor formula (31), it is sufficient to show that the function $g_{\tilde{\theta}}:[0, t] \times \mathbb{Z}_{M \geqslant}^{k} \rightarrow \mathbb{R}$ defined by $g_{\tilde{\theta}}(s, \theta)=\operatorname{det}\left(p_{t-s}\left(\theta_{i}, \tilde{\theta}_{j}\right)\right)$ is the unique solution to the following equation equipped with terminal and boundary conditions:

$$
\begin{aligned}
& \left(\partial_{s}+L\right) g_{\tilde{\theta}}=0, \\
& \left.g_{\tilde{\theta}}\right|_{[0, t] \times \partial \mathbb{Z}_{M \geqslant}^{k}}=0, \\
& g_{\tilde{\theta}}(t, \theta)=\delta_{\tilde{\theta}}(\theta) .
\end{aligned}
$$

One checks easily that $g_{\tilde{\theta}}$ is a solution of (33). In order to check that the solution is unique, one needs to prove a maximum principle for this equation, but this is easily done by mimicking the standard proof for parabolic equations.

We now show that $g_{\tilde{\theta}}(0, \theta)$ gives the desired probability. Let $N(s)=\left(N_{1}(s), \ldots, N_{k}(s)\right)$ be the Poisson process on $\mathbb{Z}_{M \geqslant}^{k}$ with initial condition $N(0)=\left(\theta_{1}, \ldots, \theta_{k}\right)$. Let $\tau=\inf \{s \in$ $\left.[0, t]: N(s) \in \partial \mathbb{Z}_{M \geqslant}^{k}\right\}$. By Ito's formula,

$$
g_{\tilde{\theta}}(s, N(s \wedge \tau))-g_{\tilde{\theta}}(0, \theta)-\int_{0}^{s \wedge \tau}\left(\partial_{r}+L\right) g_{\tilde{\theta}}(r, N(r)) \mathrm{d} r
$$

is a martingale. Taking expectations, we obtain

$$
g_{\tilde{\theta}}(0, \theta)=\mathbb{E}^{\theta} g(s, N(s \wedge \tau)) \quad \text { for } \quad s \in[0, t] .
$$

Letting $s=t$, (37) becomes $g_{\tilde{\theta}}(0, \theta)=\mathbb{E}^{\theta} g(t, N(t \wedge \tau))$. Using the definition of $\tau$ and the terminal condition (35), we see that

$$
g_{\tilde{\theta}}(0, \theta)=\mathbb{P}^{\theta}\left(t<\tau \text { and } N_{t}=\tilde{\theta}\right),
$$

which proves (31). Thus, for buses starting at locations $0 \leqslant \theta_{1}<\cdots<\theta_{k} \leqslant M-1$ at time $t=0$, we have

Prob(buses are at locations $0 \leqslant \tilde{\theta}_{1}<\cdots \tilde{\theta}_{k} \leqslant M-1$ at time $t$; no intersection for all 0

$$
\leqslant s \leqslant t)=\mathbb{P}(t<\tau \text { and } N(t)=\tilde{\theta})=g_{\tilde{\theta}}(0, \theta)=\operatorname{det}\left(p_{t}\left(\theta_{i}, \tilde{\theta}_{j}\right)\right)_{i, j=1}^{k},
$$

as desired.

This formula enables us to obtain, in particular, a Karlin-McGregor-type formula for the solution of the following natural problem for the buses on a circular bus route. Imagine that the initial locations of the buses are at $\theta_{i}=i-1$ for $i=1, \ldots, k$. Suppose that the buses return to these locations at some fixed time $T$ later. For any time $0<t<T$, it immediately follows from the formula above and the Markov property that the distribution, $Q_{t}\left(\tilde{\theta}_{1}, \ldots, \tilde{\theta}_{k}\right)$, of the locations of the buses conditioned on arriving at $\theta_{1}, \ldots, \theta_{k}$ at time $T$, is given by the formula,

$$
Q_{t}\left(\tilde{\theta}_{1}, \ldots, \tilde{\theta}_{k}\right)=\frac{\operatorname{det}\left(p_{t}\left(\theta_{i}, \tilde{\theta}_{j}\right) \operatorname{det}\left(p_{T-t}\left(\tilde{\theta}_{i}, \theta_{j}\right)\right)\right.}{\operatorname{det}\left(p_{T}\left(\theta_{i}, \theta_{j}\right)\right.},
$$

into which we may now substitute (31). Observe that the number of rotations about the circle is not fixed. We plan to investigate the asymptotic behaviour of this model in a future paper.

The circular bus route has some overlap with [9] which studies viscous walkers on the discrete circle. A useful recent review of the viscous walker problem is given in [12]. 


\section{Acknowledgments}

The authors would like to thank Y Avron for bringing the work of Krbálek and Šeba to their attention. The authors would like to thank P Šeba and E Bachmat for providing useful information. The authors would also like to thank the anonymous referees for pointing out $[9,12]$ and improving the presentation. The work of the authors was supported in part by NSF grants DMS0457335, DMS0402047, DMS0296084/0500923 and DMS0202530/0553403, respectively.

\section{References}

[1] Abramowitz M and Stegun I (ed) 1992 Handbook of Mathematical Functions with Formulas, Graphs, and Mathematical Tables (New York: Dover) (reprint of the 1972 edition)

[2] Ben Hough J, Krishnapur M, Peres Y and Virag B 2005 Determinantal processes and independence Preprint arxiv.org/abs/math.PR/0503110

[3] Borodin A and Olshanski G 2004 Markov processes on partitions Preprint arxiv.org/abs/math/0409075 (Prob. Th. Rel. Fields at press)

[4] Borodin A and Rains E M 2005 Eynard-Mehta theorem, Schur process, and their Pfaffian analogs J. Stat. Phys. 121 (3-4) 291-317 (Preprint arxiv.org/abs/math-ph/0409059)

[5] Deift P, Kriecherbauer T, McLaughlin K T-R, Venakides S and Zhou X 1999 Strong asymptotics of orthogonal polynomials with respect to exponential weights Commun. Pure Appl. Math. 52 1491-552

[6] Deift P, Kriecherbauer T, McLaughlin K T R, Venakides S and Zhou X 1999 Uniform asymptotics for polynomials orthogonal with respect to varying exponential weights and applications to universality questions in random matrix theory Commun. Pure Appl. Math. 52 1335-425

[7] Deift P A 1999 Orthogonal Polynomials and Random Matrices: A Riemann-Hilbert Approach (Courant Lecture Notes in Mathematics vol 3) (New York: New York University Courant Institute of Mathematical Sciences)

[8] Eynard B and Mehta M 1998 Matrices coupled in a chain: I. Eigenvalue correlations J. Phys. A: Math. Gen. 31 4449-56

[9] Fulmek M 2003 Nonintersecting lattice paths on the cylinder Preprint arxiv.org/abs/math-co/0311331

[10] Johansson K 2002 Non-intersecting paths, random tilings and random matrices Probab. Theory Relat. Fields 123 225-80

[11] Karlin S and McGregor J 1959 Coincidence probabilities Pacific J. Math. 9 1141-64

[12] Katori M and Tanemura H 2005 Nonintersecting paths, noncolliding diffusion processes, and representation theory Preprint arxiv.org/abs/math-pr/0501218

[13] König W and O'Connell N 2001 Eigenvalues of the laguerre process as non-colliding squared bessel processes Electron. Commun. Probab. 6 107-14 (electronic)

[14] König W, O’Connell N and Roch S 2002 Non-colliding random walks, tandem queues, and discrete orthogonal polynomial ensembles Electron. J. Probab. 724 (electronic)

[15] Krbálek M and Šeba P 2000 Statistical properties of the city transport in Cuernavaca (Mexico) and random matrix theory J. Phys. A: Math. Gen. 33 229-34

[16] Krbálek M and Šeba P 2003 Headway statistics of public transport in Mexican cities J. Phys. A: Math. Gen. 36 L7-L11

[17] Kuijlaars A B J and Vanlessen M 2002 Universality for eigenvalue correlations from the modified Jacobi unitary ensemble Int. Math. Res. Not. 1575-600

[18] Mehta M 2004 Random matrices Pure and Applied Mathematics (Amsterdam) vol 142, 3rd edn (Amsterdam: Elsevier/Academic)

[19] Nagao T and Wadati M 1991 Correlation functions of random matrix ensembles related to classical orthogonal polynomials J. Phys. Soc. Japan $603298-322$

[20] Saff E and Totik V 1997 Logarithmic potentials with external fields Grundlehren der Mathematischen Wissenschaften Fundamental Principles of Mathematical Sciences vol 316 (Berlin: Springer) (appendix B by Thomas Bloom)

[21] Soshnikov A 2000 Determinantal random point fields Usp. Mat. Nauk 55 107-60

[22] Tracy C and Widom H 1998 Correlation functions, cluster functions, and spacing distributions for random matrices J. Stat. Phys. 92 809-35 